\title{
Interactive Rough-Granular Computing in Pattern Recognition
}

\author{
Andrzej Skowron ${ }^{1}$, Jan Bazan ${ }^{2}$, and Marcin Wojnarski ${ }^{3}$ \\ 1 Institute of Mathematics, Warsaw Unvisersity \\ Banacha 2, 02-097 Warsaw, Poland \\ skowron@mimuw.edu.pl \\ 2 Chair of Computer Science, University of Rzeszów \\ Rejtana 16A, 35-310 Rzeszów, Poland \\ bazan@univ.rzeszow.pl \\ 3 Faculty of Mathematics, Informatics and Mechanics, Warsaw University \\ Banacha 2, 02-097 Warsaw, Poland \\ mwojnars@ns. onet.pl
}

\begin{abstract}
We discuss the role of generalized approximation spaces and operations on approximation spaces in searching for relevant patterns. The approach is based on interactive rough-granular computing (IRGC) in the WisTech program. We also present results on approximation of complex vague concepts in real-life projects from different domains using the approach based on ontology approximation. Software projects supporting IRGC are reported.
\end{abstract}

\section{Introduction}

Approximation spaces belong to the broad spectrum of basic subjects investigated in rough set theory (see, e.g., 2/37/3202122 ). Over the years different aspects of approximation spaces were investigated and many generalizations of the approach based on Z. Pawlak's indiscernibility equivalence relation [10 11] were proposed.

We discuss some aspects of approximation spaces in the framework of IRGC 1223 . These aspects are important from an application point of view, e.g., in searching for approximation of complex concepts (see, e.g., 12 and the bibliography in [1]). Two kinds of interactions in searching for relevant approximation spaces are distinguished. The first one is related to attributes which are interpreted as sensors making it possible to store the results of interaction with the environments. The second ones are resulting in constructions of new approximation spaces from already constructed ones. This necessitates some refinements of basic concepts such as signatures of objects, attributes as well as the semantics of what are known as granular formulas. Signatures of objects are mapped to granular formulas constructed over some generic formulas. In this way, we obtain constructive descriptions of granular neighborhoods of objects defined by granular formulas. The constructive definitions of granular neighborhoods also make it possible to define neighborhood size in terms of the size of a granular

S. Chaudhury et al. (Eds.): PReMI 2009, LNCS 5909, pp. $92-972009$.

(C) Springer-Verlag Berlin Heidelberg 2009 
formula that defines the granular neighborhood. We present a generalization of uncertainty functions assigning granular neighborhoods to objects. The neighborhoods considered so far [20] were treated as subsets of objects. However, in applications these neighborhoods consist of structural objects of different types, often of high order (see, e.g., 6] ). These types can be interpreted as structural schemes of perceived complex objects. For example, in the discovery of patterns in spatio-temporal reasoning from data, structural schemes may correspond to types of indiscernibility classes, time widows or their clusters, sequences of time widows or their clusters, sets of sequences of time widows representing processes and their interactions. Granular neighborhoods are considered in these more general cases as models of neighborhoods of higher order structural objects. We also generalize rough inclusion functions. The approximation space definition is included in Section 2. We consider approximation operations as components of approximation spaces. This is due to the fact that, in general, approximation operations may be defined in many different ways, e.g., in the case of coverings 311]. Together with approximation spaces we also consider the quality measure of approximation spaces. Approximation spaces are parameterized and their parameters are related to all components of approximation spaces including uncertainty functions, language of granular formulas, rough inclusion functions, approximation operations. Optimization of parameters is based on searching for (semi)optimal approximation space, i.e., for an approximation space for which the chosen quality measure takes (semi)optimal value on the set of objects under consideration. We propose to use the quality measures based on a variation of the minimal description length principle (MDL) 14. The discussed issues are important in solving different data mining tasks, (e.g., in searching for approximation of complex concepts [1] or in process mining [8]). Finally, software systems supporting IRGC in our projects are reported.

\section{Approximation Spaces}

Approximation spaces can be treated as granules used for concept approximation. They are some special parameterized relational structures. Tuning of parameters makes it possible to search for relevant approximation spaces relative to given concepts. In this section, we discuss a generalization of definition of approximation space from [20]) introduced in 22 .

Lt us assume $P_{\omega}\left(U^{*}\right)=\bigcup_{i \geq 1} P^{i}\left(U^{*}\right)$, where $P^{1}\left(U^{*}\right)=P\left(U^{*}\right)$ and $P^{i+1}\left(U^{*}\right)=$ $\left.P\left(P^{i}\left(U^{*}\right)\right)\right)$ for $i \geq 1$. If $X \in P_{\omega}\left(U^{*}\right)$ and $U \subseteq U^{*}$ then by $X \uparrow U$ we denote the set defined as follows (i) if $X \in P\left(U^{*}\right)$ then $X \uparrow U=X \cap U$ and (ii) for any $i \geq 1$ if $X \in P^{i+1}\left(U^{*}\right)$ then $X \uparrow U=\{Y \uparrow U: Y \in X\}$.

Definition 1. An approximation space over a set of attributes $A$ is a system

$$
A S=(U, L, I, \nu, L O W, U P P)
$$

where

- $U$ is a sample of objects with known signatures relative to a given set of attributes $A$, 
- L is a language of granular formulas defined over atomic formulas corresponding to generic formulas from signatures [22],

- $I: U^{*} \rightarrow P_{\omega}\left(U^{*}\right)$ is an uncertainty function, where $U^{*} \supseteq U$ and the set $U^{*}$ is such that for any object $u \in U^{*}$ the signature $I n f_{A}(u)$ of $u$ relative to $A$ can be obtained (as the result of sensory measurements on $u$ ); we assume that the granular neighborhood $I(u)$ is defined by $\operatorname{Inf}_{A}(u)$, i.e., $I(u)$ is defined by a granular formula $\alpha$ selected from $L$ by $\operatorname{Inf}_{A}(u)$ [22],

$-\nu: P_{\omega}\left(U^{*}\right) \times P_{\omega}\left(U^{*}\right) \rightarrow[0,1]$ is a rough inclusion function,

- LOW and UPP are the lower approximation operation and the upper approximation operation, respectively, defined on elements from $P_{\omega}\left(U^{*}\right)$ with values in $P_{\omega}\left(U^{*}\right)$ such that

1. $\nu(L O W(X), U P P(X))=1$ for any $X \in P_{\omega}\left(U^{*}\right)$,

2. $L O W(X) \uparrow U$ is included in $X \uparrow U$ to a degree at least deg, i.e., $\nu(L O W(X) \uparrow U, X \uparrow U)) \geq \operatorname{deg}$ for any $X \in P_{\omega}\left(U^{*}\right)$,

3. $X \uparrow U$ is included in $U P P(X) \uparrow U$ to a degree at least deg, i.e., $\nu(X \uparrow$ $U, U P P(X) \uparrow U) \geq$ deg for any $X \in P_{\omega}\left(U^{*}\right)$,

where deg is a given threshold from the interval $[0,1]$.

Figure 1 illustrates this computation process of the granular formulas and the granular neighborhoods by means of granular formulas.

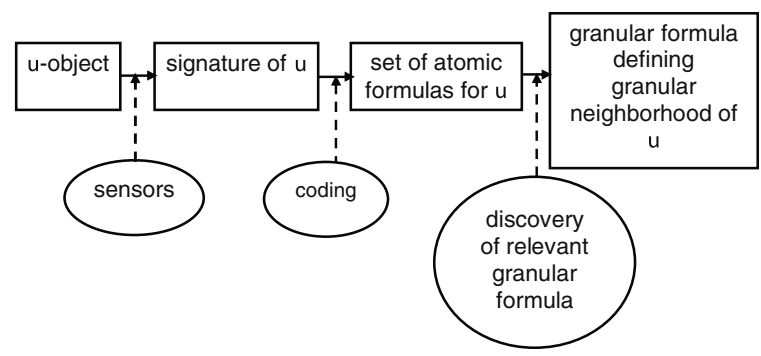

Fig. 1. From objects to granular neighborhoods

Note that this satisfiability is defined for all objects from $U^{*}$, not only for objects from a sample $U \subseteq U^{*}$. Moreover, in computation of $I(u)$ for $u \in U^{*}$ can be used not only the signature of the object $u$ currently perceived but also already known signatures of some other objects from a sample $U$ as well as higher level knowledge previously induced or acquired from experts. The details explaining how granular formulas are hierarchically constructed will be explained elsewhere. Here, we only give an illustrative example. Let us assume that granular formulas on the hierarchical level $j$ were defined. They can be treated as objects of an information system. For example, they can represent time windows. In this case, they are equal to the sets of pairs $\left(\alpha_{i}, \alpha_{i+1}\right)$ of formulas, for $i=1, \ldots, T-1$, where $T$ is the time window length and each $\alpha_{i}$ is the conjunction of descriptors (atomic formulas) for the object observed at time corresponding to $i$. Next, formulas from a new language are used for defining properties of such granular formulas (e.g., analogously to indiscernibility classes of information systems). 
In our example, with time windows, formulas from such a language describe properties of time windows. The defined sets are granular formulas on the level $j+1$. Let us consider some illustrative examples explaining how can be defined the semantics of granular formulas. If $\alpha$ is an atomic formula than $\|\alpha\|_{U^{*}}=\{u \in$ $\left.U^{*}: u \models \alpha\right\}$. If $\alpha \in L_{0}$ then $\|\alpha\|_{U^{*}}$ is defined using semantics of connectives selected for construction of formulas from $L_{0}$. If $\|\alpha\|_{U^{*}}$ is defined for $\alpha \in L$ for some language of granular formulas and $\beta \in P(L)$ then $\|\beta\|_{U^{*}}=\left\{\|\alpha\|_{U^{*}}: \alpha \in\right.$ $\beta\}$. However, one can also assume $\|\beta\|_{U^{*}}=\bigcup\left\{\|\alpha\|_{U^{*}}: \alpha \in \beta\right\}$. In the latter case, the defined granular neighborhood is from $P\left(U^{*}\right)$ but information on semantics of the granular neighborhood components are lost.

Approximation spaces are parameterized and their parameters are related to all components of approximation spaces including uncertainty functions, language of granular formulas, rough inclusion functions, approximation operations. In this way, we obtain a family $A P P R O X_{-} S P A C E S$ of approximation spaces corresponding to different values of parameters. Optimization of parameters is based on searching for the (semi)optimal approximation space, i.e., for an approximation space with the (semi)optimal value of the quality measure on the set of objects under consideration. For the given family APPROX_SPACES, a quality measure $Q$ is a function which assigns to any $A S \in A P P R O X \_S P A C E S$ and $X \in P_{\omega}\left(U^{*}\right)$ a nonnegative number $Q(A S, X)$ called an approximation quality of $X$ in $A S \mathbb{1}$. For a given triple (APPROX_SPACES, Q, X), where $X \subseteq U^{*}$, we consider the optimization problem. This is the searching problem for (semi)optimal approximation space $A S_{0} \in A P P R O X_{-} S P A C E S$ such that $Q\left(A S_{0}, X\right)=\inf \left\{Q(A S, X): A S \in A P P R O X_{-} S P A C E S\right\}$. We use quality measures based on different versions of the minimal length principle [14.

\section{Software Systems}

In this section, we present a short information about two software platforms Rough Set Interactive Classification Engine (RoughICE) 15] and TunedIT [19] which are supporting our projects based on IRGC.

RoughICE is a software platform supporting the approximation of spatiotemporal complex concepts in the given concept ontology acquired in the dialogue with the user. RoughICE is freely available on the web side [15. The underlying algorithmic methods, especially for generating reducts and rules, discretization and decomposition, are outgrows of our previous tools such as RSES [16] and RSESlib [17. RoughICE software and underlying computational methods have been successfully applied in different data mining projects (e.g., in mining traffic data and medical data; for details see [1] and the literature cited in [1]).

TunedIT platform, launched recently by members of our research group, facilitates sharing, evaluation and comparison of data-mining and machine-learning algorithms. The resources used in our experiments - algorithms and datasets in particular - will be shared on TunedIT website. This website already contains

\footnotetext{
${ }^{1}$ In the case of classification $X \in P^{2}\left(U^{*}\right)$ and $X$ is a partition of $U^{*}$.
} 
many publicly available datasets and algorithms, as well as performance data for nearly 100 algorithms tested on numerous datasets - these include the algorithms from Weka, Rseslib libraries, and the datasets from UCI Machine Learning Repository. Everyone can contribute new resources and results. TunedIT is composed of three complementary modules: TunedTester, Repository and Knowledge Base. TunedIT may help researchers design repeatable experiments and generate reproducible results. It may be particularly useful when conducting experiments intended for publication, as reproducibility of experimental results is the essential factor that determines research value of the paper. TunedIT helps also in dissemination of new ideas and findings. Every researcher may upload his implementations, datasets and documents into Repository, so that other users can find them easily and employ in their own research.

For more details on RoughICE and TunedIT the reader is referred to [15] and [19], respectively.

\section{Conclusions}

We discussed a generalization of approximation spaces based on granular formulas and neighborhoods. Efficient searching strategies for relevant approximation spaces are crucial for application (e.g., in searching for approximation of complex concepts or in process mining). Some of such strategies based on ontology approximation were already elaborated and implemented for solving real-life problems (see, e.g., [1]). We are working on the project aiming at developing new strategies for hierarchical modeling as well as methods based on adaptive interactive granular computations 5] for selection and construction of features. These features are next used as relevant components of approximation spaces, in particular granular neighborhoods of approximation spaces.

\section{Acknowledgements}

The research has been partially supported by the grants N N516 368334 and N N516 077837 from Ministry of Science and Higher Education of the Republic of Poland.

\section{References}

1. Bazan, J.G.: Hierarchical classifiers for complex spatio-temporal concepts. In: Peters, J.F., Skowron, A., Rybiński, H. (eds.) Transactions on Rough Sets IX. LNCS, vol. 5390, pp. 474-750. Springer, Heidelberg (2008)

2. Bazan, J., Skowron, A., Swiniarski, R.: Rough sets and vague concept approximation: From sample approximation to adaptive learning. In: Peters, J.F., Skowron, A. (eds.) Transactions on Rough Sets V. LNCS, vol. 4100, pp. 39-62. Springer, Heidelberg (2006)

3. Grzymała-Busse, J., Rząsa, W.: Local and global approximations for incomplete data. In: Peters, J.F., Skowron, A. (eds.) Transactions on Rough Sets VIII. LNCS, vol. 5084, pp. 21-34. Springer, Heidelberg (2008) 
4. Hastie, T., Tibshirani, R., Friedman, J.H.: The Elements of Statistical Learning: Data Mining, Inference, and Prediction, 2nd edn. Springer, Heidelberg (2008)

5. Jankowski, A., Skowron, A.: Logic for artificial intelligence: The Rasiowa-Pawlak school perspective. In: Ehrenfeucht, A., Marek, V., Srebrny, M. (eds.) Andrzej Mostowski and Foundational Studies, pp. 106-143. IOS Press, Amsterdam (2008)

6. Ng, K.S., Lloyd, J.W., Uther, W.T.B.: Probabilistic modelling, inference and learning using logical theories. Annals of Mathematics and Artificial Intelligence 54(1-3), 159-205 (2008)

7. Nguyen, H.S.: Approximate Boolean Reasoning: Foundations and Applications in Data Mining. In: Peters, J.F., Skowron, A. (eds.) Transactions on Rough Sets V. LNCS, vol. 4100, pp. 344-523. Springer, Heidelberg (2006)

8. Nguyen, H.S., Jankowski, A., Skowron, A., Stepaniuk, J., Szczuka, M.: Discovery of process models from data and domain knowledge: A rough-granular approach. In: Yao, J.T. (ed.) Novel Developments in Granular Computing: Applications for Advanced Human Reasoning and Soft Computation, IGI Global, Hershey (accepted)

9. Pal, S.K., Shankar, B.U., Mitra, P.: Granular computing, rough entropy and object extraction. Pattern Recognition Letters 26(16), 2509-2517 (2005)

10. Pawlak, Z.: Rough Sets: Theoretical Aspects of Reasoning about Data, System Theory, Knowledge Engineering and Problem Solving, vol. 9. Kluwer Academic Publishers, Dordrecht (1991)

11. Pawlak, Z., Skowron, A.: Rudiments of rough sets. Information Sciences 177(1), 3-27 (2007); Rough sets: Some extensions. Information Sciences 177(1), 28-40

12. Pedrycz, W., Skowron, A., Kreinovich, V. (eds.): Handbook of Granular Computing. John Wiley \& Sons, New York (2008)

13. Peters, J., Henry, C.: Reinforcement learning with approximation spaces. Fundamenta Informaticae 71(2,3), 323-349 (2006)

14. Rissanen, J.: Modeling by shortest data description. Automatica 14, 465-471 (1978)

15. The Rough Set Interactive Classificstion Engine (RoughICE), http://logic.mimuw.edu.pl/ bazan/roughice

16. The Rough Set Exploration System (RSES), http://logic.mimuw.edu.pl/ rses

17. The RSES-lib project, http://rsproject.mimuw.edu.pl

18. The road simulator, http://logic.mimuw.edu.pl/ bazan/simulator

19. The TunedIT platform, http://tunedit.org/

20. Skowron, A., Stepaniuk, J.: Tolerance approximation spaces. Fundamenta Informaticae 27, 245-253 (1996)

21. Skowron, A., Stepaniuk, J., Peters, J., Swiniarski, R.: Calculi of approximation spaces. Fundamenta Informaticae 72(1-3), 363-378 (2006)

22. Skowron, A., Stepaniuk, J., Peters, J.F., Swiniarski, R.: Approximation spaces revisited. In: Proceedings of the Concurrency, Specification \& Programming 2009 (CS\&P 2009), Przegorzały, Kraków, Poland, September 28-30, pp. 538-549. Warsaw University (2009)

23. Skowron, A., Szczuka, M.: Toward interactive computations: A rough-granular approach. In: Koronacki, J., Wierzchon, S.T., Ras, Z.W., Kacprzyk, J. (eds.) Advances in Machine learning II, Dedicated to the memory of Ryszard Michalski. Studies in Computational Intelligence, vol. 263, pp. 1-20. Springer, Heidelberg (2009) (in print)

24. Ziarko, W.: Variable precision rough set model. Journal of Computer and System Sciences 46, 39-59 (1993) 